\title{
Epothilone D Improves Microtubule Density, Axonal Integrity, and Cognition in a Transgenic Mouse Model of Tauopathy
}

\author{
Kurt R. Brunden, ${ }^{1 *}$ Bin Zhang, ${ }^{1 *}$ Jenna Carroll, ${ }^{1}$ Yuemang Yao, ${ }^{1}$ Justin S. Potuzak, ${ }^{2}$ Anne-Marie L. Hogan, ${ }^{2}$ \\ Michiyo Iba, ${ }^{1}$ Michael J. James, ${ }^{1}$ Sharon X. Xie, ${ }^{3,4}$ Carlo Ballatore, ${ }^{1,2}$ Amos B. Smith III, ${ }^{2}$ Virginia M.-Y. Lee, ${ }^{1}$ \\ and John Q. Trojanowski ${ }^{1,3}$ \\ ${ }^{1}$ Center for Neurodegenerative Disease Research, Institute on Aging, Department of Pathology and Laboratory Medicine, School of Medicine, ${ }^{2}$ Department \\ of Chemistry, School of Arts and Sciences, ${ }^{3}$ Institute on Aging, and ${ }^{4}$ Department of Biostatistics and Epidemiology, University of Pennsylvania, \\ Philadelphia, Pennsylvania 19104
}

Neurons in the brains of those with Alzheimer's disease (AD) and many frontotemporal dementias (FTDs) contain neurofibrillary tangles comprised of hyperphosphorylated tau protein. Tau normally stabilizes microtubules (MTs), and tau misfolding could lead to a loss of this function with consequent MT destabilization and neuronal dysfunction. Accordingly, a possible therapeutic strategy for AD and related "tauopathies" is treatment with a MT-stabilizing anti-cancer drug such as paclitaxel. However, paclitaxel and related taxanes have poor blood-brain barrier permeability and thus are unsuitable for diseases of the brain. We demonstrate here that the MT-stabilizing agent, epothilone D (EpoD), is brain-penetrant and we subsequently evaluated whether EpoD can compensate for tau loss-of-function in PS19 tau transgenic mice that develop forebrain tau inclusions, axonal degeneration and MT deficits. Treatment of 3-month-old male PS19 mice with low doses of EpoD once weekly for a 3 month period significantly improved CNS MT density and axonal integrity without inducing notable side-effects. Moreover, EpoD treatment reduced cognitive deficits that were observed in the PS19 mice. These results suggest that certain brain-penetrant MT-stabilizing agents might provide a viable therapeutic strategy for the treatment of AD and FTDs.

\section{Introduction}

A feature of all neurodegenerative tauopathies, including Alzheimer's disease $(\mathrm{AD})$ and many frontotemporal dementias (FTDs), is the presence of intraneuronal neurofibrillary tangles (NFTs) formed from tau protein (V. M. Y. Lee et al., 2001). Tau normally stabilizes microtubules (MTs) (Binder et al., 1985), but tau within NFTs is hyperphosphorylated and assembled into amyloid fibrils (Kidd, 1963; V. M. Y. Lee et al., 2001). NFT density correlates with the degree of dementia in AD (Wilcock and Esiri, 1982; Arriagada et al., 1992), suggesting a neurodegenerative relationship. In fact, tau gene mutations cause neurodegenerative FTD (Goedert, 2005).

It has been postulated that tauopathies result, at least in part, from a loss of tau function (V. M. Y. Lee et al., 2001) caused by reduced MT binding of hyperphosphorylated (Alonso et al., 1994; Merrick et al., 1997) or mutant tau (Hong et al., 1998; Hasegawa et al., 1998). The resulting MT destabilization could

Received June 15, 2010; revised Aug. 20, 2010; accepted Aug. 26, 2010.

These studies were funded by the National Institutes of Health (AG24904, AG10124, AG17586, AG029213 and NS53488). We thank Xiaoyan Han for assistance with the statistical analyses.

*K.R.B. and B.Z. contributed equally to the manuscript.

Correspondence should be addressed to Dr. Kurt R. Brunden, Director of Drug Discovery, Center for Neurodegenerative Disease Research, Department of Pathology and Laboratory Medicine, University of Pennsylvania, 3600 Spruce Street, Maloney 3rd Floor, Philadelphia, PA 19104. E-mail: kbrunden@upenn.edu.

DOI:10.1523/JNEUROSCI.3059-10.2010

Copyright $\odot 2010$ the authors $\quad$ 0270-6474/10/3013861-06\$15.00/0 lead to impaired axonal transport and neuronal function, and there is, in fact, an observed reduction of stable MTs in AD brain (Hempen and Brion, 1996) and in tau transgenic (Tg) mice (Ishihara et al., 1999). Thus, known MT-stabilizing drugs such as paclitaxel might provide therapeutic benefit in tauopathies if doses could be used that avoid the side-effects observed in cancer treatment (Bedard et al., 2010). Indeed, proof-ofprinciple was obtained when paclitaxel was administered to tau Tg mice, which develop NFT-like inclusions in the brainstem and spinal cord, as drug absorption at neuromuscular junctions resulted in improved MT density and increased axonal transport in spinal motor neurons, as well as enhanced motor performance (Zhang et al., 2005). Unfortunately, paclitaxel is not suitable for treatment of true tauopathies due to poor blood-brain barrier (BBB) penetration (Fellner et al., 2002).

Epothilone D (EpoD), another known MT-stabilizing compound, has been suggested to cross the BBB (Andrieux et al., 2006) and we confirm that EpoD is brain-penetrant. We tested whether EpoD could compensate for tau loss-of-function in PS19 Tg mice, which develop tau inclusions within the forebrain (Yoshiyama et al., 2007) and, as demonstrated here, CNS MT density deficits accompanied by axonal degeneration. Treatment of 3-month-old male PS19 mice with EpoD once weekly for a 3 month period significantly improved CNS MT density and axonal integrity without inducing notable side effects. Moreover, EpoD treatment reduced cognitive deficits that developed by 6 
months of age in the PS19 mice. These data suggest that brainpenetrant MT-stabilizing agents hold promise as viable therapeutics for the treatment of tauopathies.

\section{Materials and Methods}

Synthesis of EpoD. EpoD was prepared as previously described (C. B. Lee et al., 2001; Rivkin et al., 2004). The spectroscopic properties of EpoD were identical to those reported in the literature. Compound purity was $>95 \%$ as demonstrated by LC-MS.

Pharmacokinetic analysis of plasma and brain concentrations of EpoD. Groups of mice $(n=3)$ received intraperitoneal injections of $3.7 \mathrm{mg} / \mathrm{kg}$ EpoD dissolved in $100 \%$ DMSO, followed by euthanization using University of Pennsylvania IACUC approved protocols at times ranging from 0.25 to $24 \mathrm{~h}$. In another study, groups of mice $(n=3)$ received injections of $3 \mathrm{mg} / \mathrm{kg}$ EpoD in 100\% DMSO followed by euthanization 4, 6 and $10 \mathrm{~d}$ later. The EpoD levels in brain and blood samples were determined using previously described LC-MS/MS protocols (Ballatore et al., 2010).

EpoD treatment of PS19 tau Tg mice and non-Tg littermate mice. Groups ( $n=10-13)$ of 3-month-old PS19 tau Tg mice or 3-month-old non-Tg littermates (Yoshiyama et al., 2007) were administered weekly intraperitoneal injections of $1 \mathrm{mg} / \mathrm{kg}$ EpoD, $3 \mathrm{mg} / \mathrm{kg}$ EpoD or vehicle (DMSO), for a total of 3 months. Animals were monitored for signs of abnormal behavior or distress, and were weighed weekly. After final dosing, the mice underwent motor function and cognitive testing as described below. After euthanization, brains and optic nerve $(\mathrm{ON})$ were recovered for immunohistochemical analyses. A subset of mice from each group also underwent necropsy evaluation with organ weights recorded.

Immunohistochemical analysis of tau and phospho-tau in ON and brain slices. Untreated, EpoD-treated, or vehicle-treated mice were perfused as described previously (Yoshiyama et al., 2007). The brains and/or ON were removed and processed as described previously (Ishihara et al., 1999; Zhang et al., 2004). Sections $(6 \mu \mathrm{m})$ were stained with a rabbit anti-tau polyclonal antibody $(17026 ; 1: 10,000$ dilution) or a mouse monoclonal antibody (AT8; 1:7500 dilution) that recognizes tau phosphorylated at ser ${ }^{202 / 205}$ (Goedert et al., 1995). Secondary antibodies (Vector Laboratories) were diluted with BioGenex reagent and processed with an i600 Automatic staining system (BioGenex Laboratories). AT8positive tau pathology was rated from 0 (no positive tau pathology) to 4 (wide-spread and intense immunostaining of tau pathology) by an assessor masked to the treatment group.

Electron microscopy evaluation of ON axonal dystrophy and MT density. Transmission electron microscopy (EM) was performed on crosssectional slices of ON from PS19 mice or non-Tg littermates obtained as described above. Ultrathin sections from ON blocks were cut and mounted on EM grids and examined using a JEM1010 electron microscope (Jeol) at $80 \mathrm{kV}$. Cross sections of the entire $\mathrm{ON}$ were systematically sampled at $10,000 \times$ and $50,000 \times$, with dystrophic axons and MTs counted from multiple fields of $128 \mu \mathrm{m}$ and $0.035 \mu \mathrm{m}$, respectively, from at least 30 separate photos. The assessor was masked to the treatment group.

Complete blood counts. Blood from PS19 or non-Tg littermates treated with vehicle or EpoD was collected from the heart into MiniCollect tubes with EDTA (Greiner Bio-One) immediately before perfusion as described above. The blood samples were supplied on ice to Seventh Wave Laboratories for complete blood cell counts.

Motor testing. To assess motor coordination and balance, mice were tested on the rotarod (Med Associates). Each mouse was given an acclimation training session (four trials, $10 \mathrm{~min}$ apart) followed $1 \mathrm{~h}$ later by two test trials where the mouse was placed on the rotarod with increasing speed from 4 to $40 \mathrm{rpm}$ over $300 \mathrm{~s}$ (Dai et al., 2009). Each mouse received two consecutive trials and the mean latency to fall was recorded.

Barnes maze. Mice were tested on the Barnes maze (San Diego Instruments; model 7001-0235) to assess learning and memory (O'Leary and Brown, 2009; Patil et al., 2009). Mice were randomly assigned to different escape compartments and were allowed to habituate to the environment for $1 \mathrm{~h}$ before testing and to the maze for $30 \mathrm{~s}$ before each trial. The maze was cleaned with $70 \%$ ethanol between animals to minimize odor cues. On consecutive testing days, mice were exposed to the maze for three 2.5
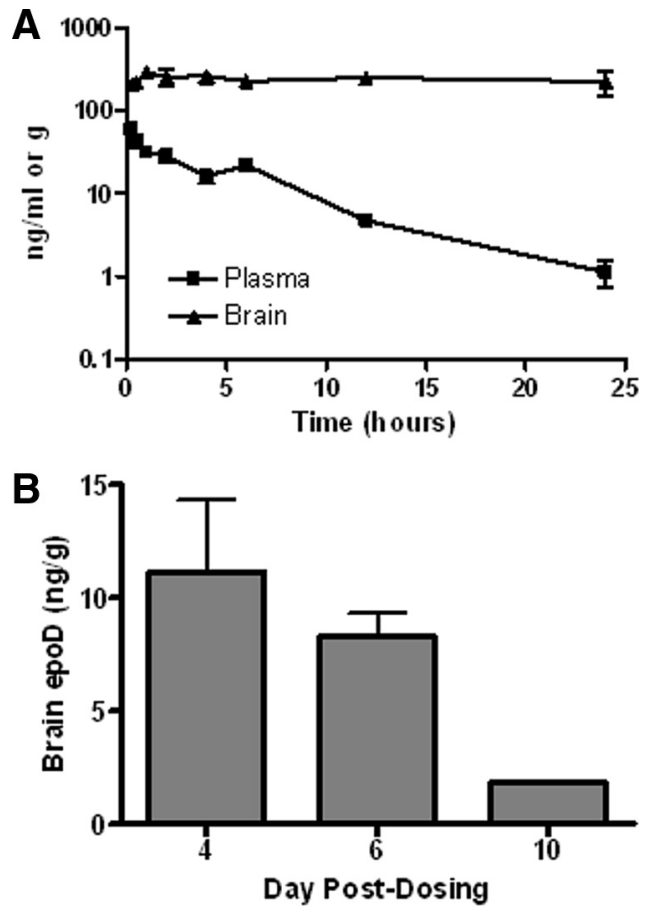

Figure 1. Pharmacokinetic testing of EpoD. $A, E p o D$ concentrations in plasma and brain were determined at multiple time points after a single intraperitoneal administration. $\boldsymbol{B}$, Brain concentrations of EpoD after an intraperitoneal dose of $3 \mathrm{mg} / \mathrm{kg}$. Error bars represent SD. Plasma and brain EpoD concentrations were significantly different $(p<0.01)$ at all times in $\boldsymbol{A}$ as determined by two-tailed $t$ tests using GraphPad Prism software with $n=3$ mice/time.

min trials that were $15 \mathrm{~min}$ apart. The first trial was not scored and mice who did not find the escape compartment were guided there and allowed to remain for $30 \mathrm{~s}$. Mice were tested for their ability to remember the fixed position of this escape compartment during trials 2 and 3, with performance scored for success rate and number of errors before finding the correct compartment (Harrison et al., 2009; Patil et al., 2009). Success rates were compared among groups using the generalized estimating equation approach (Zeger et al., 1988). The number of errors per trial were compared among groups using a linear mixed model (Laird and Ware, 1982). Statistical analyses were performed using SAS software (v9.1, SAS Institute). All statistical tests were two-sided.

\section{Results}

A therapeutic strategy for tauopathies based on a pharmacological compensation for tau loss-of-function requires a MTstabilizing compound that can readily equilibrate across the BBB. EpoD, one of several related MT-stabilizing compounds (Altmann, 2005), appears to be brain-penetrant as evidenced by its use in a mouse model of schizophrenia (Andrieux et al., 2006). To verify that EpoD can cross the $\mathrm{BBB}$, the compound was administered intraperitoneally to normal mice and plasma and brain concentrations were ascertained. EpoD concentrations in the brain exceeded plasma levels at all times after dosing and the compound was cleared more slowly from brain than plasma (Fig. $1 A)$. Subsequent testing revealed that a single dosing of EpoD (3 $\mathrm{mg} / \mathrm{kg}$ ) resulted in measurable levels of compound in the brain for up to $10 \mathrm{~d}$ (Fig. $1 \mathrm{~B}$ ). Although it is typically undesirable for a compound to be retained in the brain, this may be advantageous for a MT-stabilizing agent directed to CNS neurons, as it might allow for prolonged drug activity in the brain while minimizing sustained peripheral exposure. These results thus suggest that EpoD is suitable for proof-of-principle testing in a mouse model 

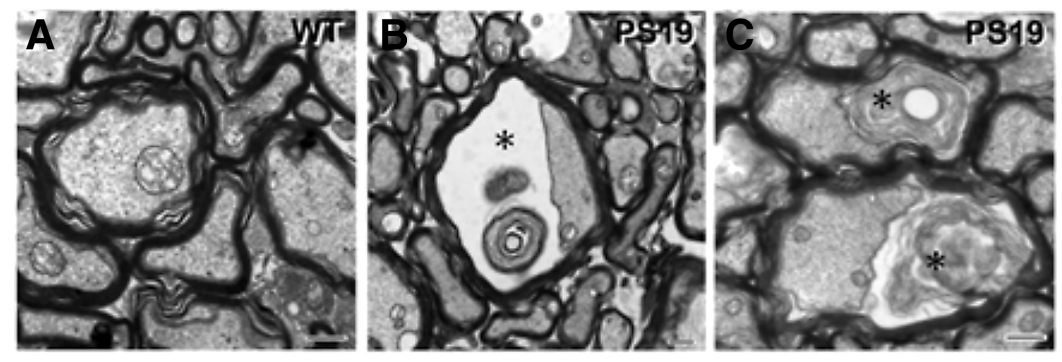

D

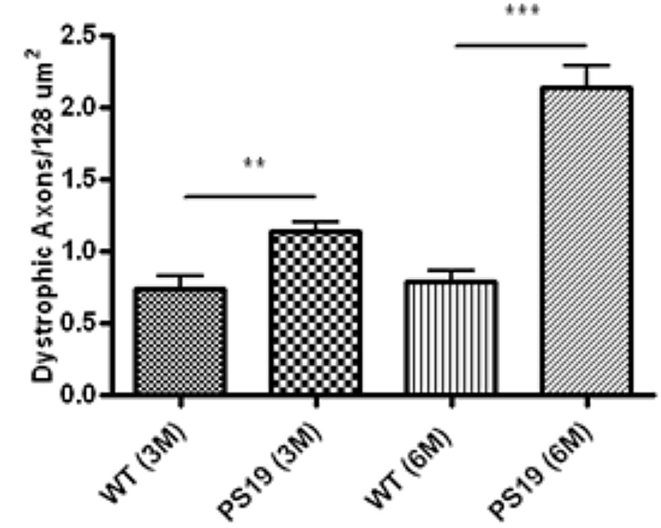

$\mathbf{E}$

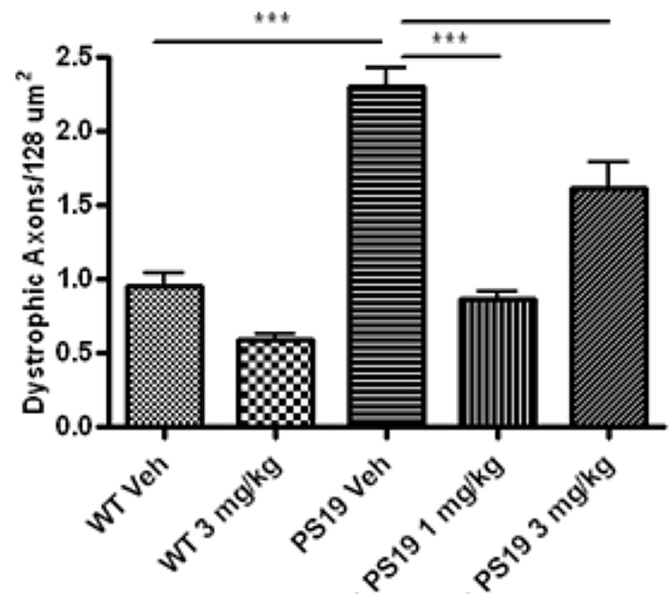

Figure 2. Axonal dystrophy in ON from PS19 mice. $A-C$, Electron micrographs of cross-sectional areas from $0 \mathrm{~N}$ at points equidistant from the globe of the eye and retina of 6-month-old WT mice (A) and PS19 mice ( $\boldsymbol{B}, \boldsymbol{C}$. $\boldsymbol{D}$, Axonal dystrophy in 0N from 3- and 6-month-old male and female PS19 mice, and non-Tg (WT) littermates. $\boldsymbol{E}$, Male WT and PS19 mice treated with EpoD or vehicle from 3 to 6 months of age were assessed for the number of dystrophic axons. Error bars represent SEM. ${ }^{* *} p<0.01,{ }^{* * *} p<$ 0.001 , as determined by two-tailed $t$ tests with $n=5-9$ mice/group for $\boldsymbol{D}$ and $n=7-8$ mice/group for $\boldsymbol{E}$.

intraperitoneal injections of vehicle or EpoD (1 mg/kg or $3 \mathrm{mg} / \mathrm{kg}$ ) for a total of 3 months. In addition, 3-month-old non-Tg littermates received $3 \mathrm{mg} / \mathrm{kg}$ EpoD or vehicle. The $3 \mathrm{mg} / \mathrm{kg}$ EpoD dose corresponds to $\sim 10 \%$ that used in a Phase II clinical study (Beer et al., 2007), which should minimize side-effects such as neutropenia that are observed with MTstabilizing drugs in human subjects (Bedard et al., 2010). PS19 and WT mice that received EpoD showed no signs of drug intolerance. Indeed, all drug-treated mice exhibited weight gain that was indistinguishable from vehicle-treated animals (supplemental Fig. $2 A$, available at www.jneurosci.org as supplemental material). Likewise, relative organ weights were similar in vehicle- and EpoD-treated mice (supplemental Table 1, available at www.jneurosci.org as supplemental material). The motor performance of EpoDtreated mice, assessed using a standard rotarod test, was not significantly different from vehicle-treated cohorts (supplemental Fig. $2 B$, available at www.jneurosci. org as supplemental material). Finally, although there was minor group-togroup variability, there were no significant differences in white blood cell counts or neutrophil content between any of the treatment cohorts (supplemental Fig. 2C,D, available at www. jneurosci.org as supplemental material). Thus, the low doses of EpoD used in these studies appeared to be well tolerated.

The effect of EpoD treatment on axonal integrity was determined by analyzing dystrophic axons in electron micrographs from $\mathrm{ON}$ of EpoD- and vehicle-treated mice. In agreement with the results of Figure 2D, 6-month-old vehicle-treated PS19 mice had an approximately twofold increase in abnormal axons relative to their WT counterparts (Fig. 2E). PS19 mice that received low-dose EpoD for 3 months showed a significant reduction in

with predominantly brain tau pathology. PS19 tau Tg mice express human tau harboring the P301S mutation (Goedert, 2005) and develop forebrain accumulations of insoluble tau, synaptic degeneration and neuron loss (Yoshiyama et al., 2007). Moreover, examination of 6-month-old PS19 mouse optic nerves (ONs), which provide readily sampled bundles of CNS axons residing behind the $\mathrm{BBB}$, reveals significant human tau expression and hyperphosphorylated tau inclusions within retinal ganglion cells from whence ON axons emanate (supplemental Fig. 1, available at www.jneurosci.org as supplemental material). Moreover, EM analysis demonstrated significantly greater numbers of dystrophic and degenerated axons in the ON of PS19 versus wildtype (WT) mice at 3 months of age, with a greater difference by 6 months of age (Fig. $2 A-D$ ).

To evaluate whether EpoD improves MT and axonal function in PS19 mice, groups of 3-month-old male PS19 mice received weekly dystrophic axons, achieving levels observed in vehicle-treated WT animals. The $3 \mathrm{mg} / \mathrm{kg}$ EpoD PS19 group also displayed significantly attenuated axonal dystrophy, although the magnitude of effect was smaller in this treatment group, perhaps due to overstabilization of MTs (Ertürk et al., 2007; Witte et al., 2008).

The ability of EpoD to influence axonal architecture was further investigated by analyzing ON MT density in the various treatment groups. As summarized in Figure 3A, the PS19 vehicle group had a lower MT density than did the WT vehicle group, providing additional evidence that tau misfolding in PS19 mice results in MT destabilization that is the likely cause of the axonal dystrophy and degeneration noted above. Notably, both doses of EpoD resulted in increased MT density in the PS19 mice, such that the values were similar to the WT vehicle group. Interestingly, EpoD also increased MT density in WT mice. 


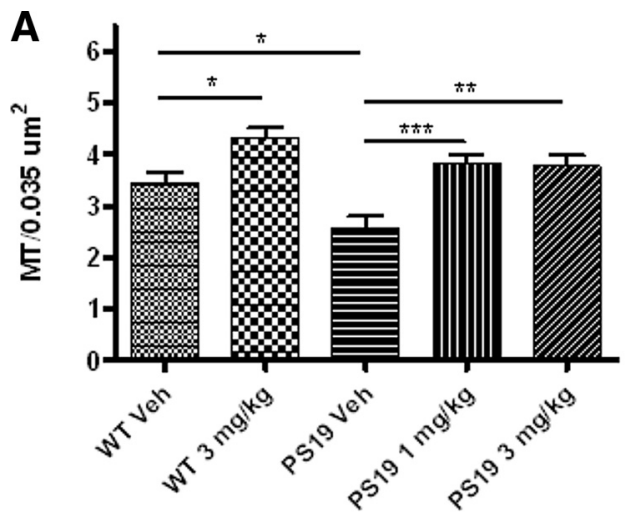

B

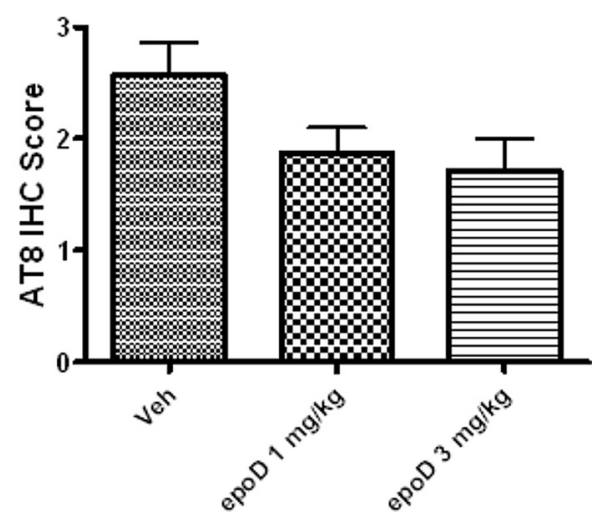

Figure 3. ON MT density and tau brain pathology in EpoD- and vehicle-treated mice. $A, M T$ density in WT and PS19 mice treated with EpoD or vehicle from 3 to 6 months of age. $\boldsymbol{B}$, Relative phospho-tau immunostaining in coronal slices of brain hemispheres. Error bars represent SEM. ${ }^{*} p<0.05,{ }^{* *} p<0.01,{ }^{* * *} p<0.001$ as determined by one-way ANOVA using a post hoc Bonferroni's multiple-comparison test with $n=7-8$ mice/group $(\boldsymbol{A}) . p>0.05$ in $\boldsymbol{B}$ as determined by Kruskal-Wallis analysis with $n=7-8$ mice/group.

PS19 mice develop tau pathology by 6 months of age in the hippocampus and amygdala (Yoshiyama et al., 2007), and we performed immunohistochemical analyses of phospho-tau in brain sections to assess whether EpoD treatment affected tau pathology (see supplemental Fig. 3, available at www.jneurosci.org as supplemental material). There was variability in the extent of tau pathology in all of the PS19 treatment groups, and while there was a trend toward lower AT8 immunostaining in the EpoDtreated mice, this did not reach statistical significance (Fig. $3 B$ ). Appreciable phospho-tau immunostaining was also still evident in ON after EpoD treatment (supplemental Fig. 1, available at www.jneurosci.org as supplemental material). These findings are not unexpected, as improving MT function and axonal integrity with a MT-stabilizing agent may not alter tau phosphorylation and aggregation.

The axonal degeneration observed in 6-month-old PS19 mice suggested that these animals might have cognitive abnormalities, and in fact it was previously demonstrated that these mice have altered long-term potentiation in the CA1 hippocampal region (Yoshiyama et al., 2007). We compared the performance of vehicle-treated PS19 mice and non-Tg littermates, as well as EpoD-treated PS19 mice, in the Barnes maze test of learning and memory (Harrison et al., 2009). Key parameters that were assessed were the success rate at identifying an escape hole within the maze and the number of errors made in each trial before identifying the proper target. As illustrated in Figure 4, vehicletreated PS19 mice displayed significantly poorer performance in both measures relative to vehicle-treated WT mice. All of the
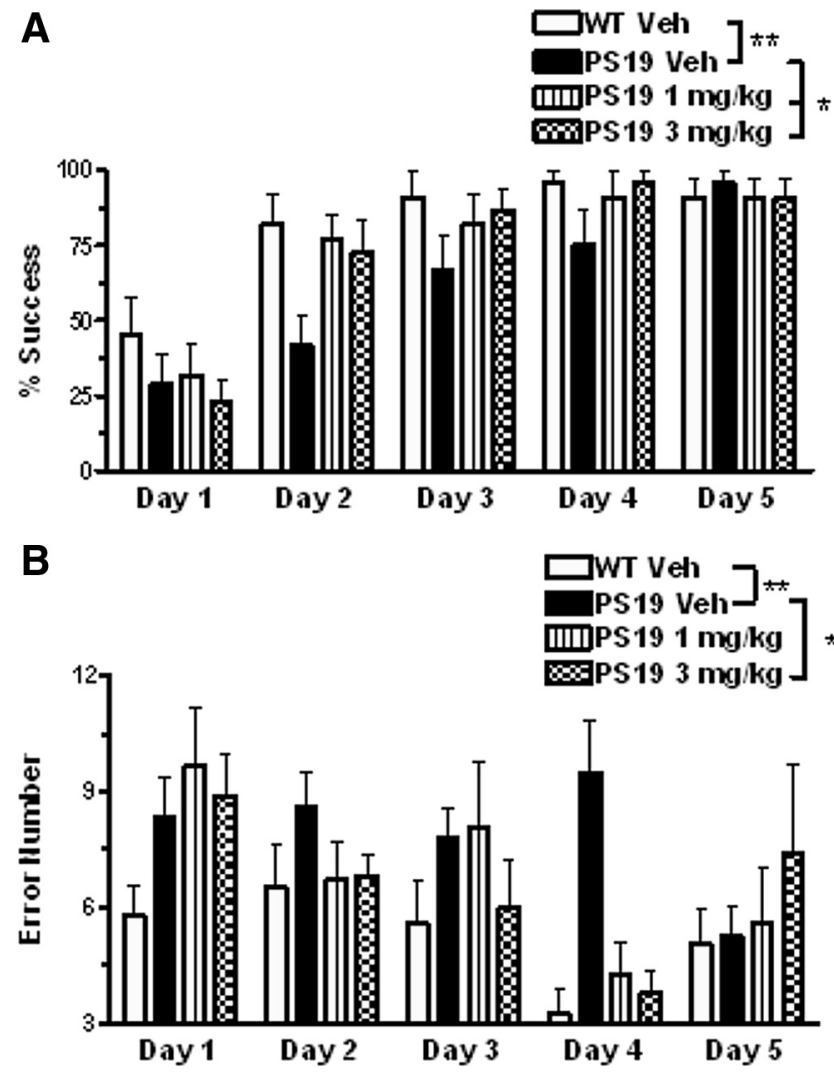

Figure 4. Barnes maze performance of WT and PS19 mice treated with EpoD or vehicle. $\boldsymbol{A}, \boldsymbol{B}$ Percentage of trials that were successfully completed $(\boldsymbol{A})$ and the number of errors per trial $(\boldsymbol{B})$. Error bars represent SEM. ${ }^{*} p<0.05,{ }^{* *} p<0.01$ as determined by a repeated-measures statistical analysis with $n=10-12$ mice/group.

PS19 groups performed worse than the non-Tg vehicle group on both measures during the first day of testing. However, by the second day of testing the EpoD-treated PS19 mice were significantly improved in both endpoints compared with the PS19 vehicle group, attaining performance that was comparable to WT vehicle animals. From the third day onward, the percentage of successful trials for the vehicle-treated PS19 mice began to approach that of the WT mice, and all treatment groups reached nearly $100 \%$ success by day 5 (Fig. $4 A$ ). Nonetheless, both EpoD dose groups also showed a trend toward improved success on days 3 and 4, and there was an overall statistically significant improvement for both EpoD groups relative to vehicle-treated PS19 mice. There was also a trend for the EpoD-treated mice to have fewer errors than the vehicle-treated mice on days 2-4 (Fig. $4 B$ ), with the EpoD high-dose group showing an overall improvement in error number compared with the PS19 vehicle group. The $1 \mathrm{mg} / \mathrm{kg}$ EpoD cohort did not reach significance, largely due to poor performance on the third day of testing. In summary, EpoD stabilization of MTs appears to result in improvement of cognitive performance in the PS19 mice.

\section{Discussion}

Although there is uncertainty as to how tau alterations in $\mathrm{AD}$ and other tauopathies lead to neuronal damage, one hypothesis that has gained credence is that a loss of tau function results in MT destabilization with consequent impairment of axonal transport. Of particular note are data which suggest that small molecule (Zhang et al., 2005) or peptide (Matsuoka et al., 2007) MTstabilizing agents can improve pathological and functional out- 
comes in Tg mouse models with NFT-like tau inclusions. Although these data provided critical proof-of-principle, the only small molecule MT-stabilizing agent that has been evaluated in a Tg mouse tauopathy model is paclitaxel (Zhang et al., 2005), which does not cross the BBB significantly and acted through uptake at neuromuscular junctions in this mouse model. Thus, there has been no demonstration of a small molecule MTstabilizing compound compensating for a loss of tau function after crossing the BBB. We found that EpoD readily crosses the $\mathrm{BBB}$ and is cleared more slowly from brain than plasma, with measurable levels in brain up to $10 \mathrm{~d}$ after a single $3 \mathrm{mg} / \mathrm{kg}$ i.p. administration.

EpoD was administered once weekly for 3 months to 3-month-old PS19 tau Tg mice that have minimal tau pathology but which by 6 months of age develop forebrain NFT-like deposits, as well as axonal MT loss and axonal degeneration in the CNS. This dosing paradigm examined the effects of EpoD during the onset of tau pathology, in accordance with the growing evidence that the development of tau pathology in AD continues after the onset of clinical symptoms (Jack et al., 2010). Thus, the PS19 mice were administered EpoD at what might be the equivalent of the prodromal stage of AD or mild cognitive impairment (Jack et al., 2010). The low doses of EpoD used here did not affect body weight or induce behavioral impairments, nor was there evidence of neutropenia, a common side-effect of MT-stabilizing drugs (Bedard et al., 2010).

The ON axonal dystrophy observed in 6-month-old PS19 mice was significantly attenuated by the 3 month treatment with EpoD. Moreover, both doses of EpoD corrected the ON MT density deficit seen in 6-month-old PS19 mice, resulting in MT densities that were similar to those of non-Tg littermates. Notably, EpoD treatment also improved cognitive performance in the 6-month-old PS19 mice that were found to have a learning and memory deficit when tested in the Barnes maze (Harrison et al., 2009). Both doses of EpoD led to significant improvements in the percentage of successful Barnes maze trials upon repeated testing. Furthermore, EpoD also led to a reduction in the number of errors in the Barnes maze, although only the $3 \mathrm{mg} / \mathrm{kg}$ dose reached statistical significance.

Although EpoD led to improvements in CNS axonal integrity and MT content, as well as in cognitive performance, in PS19 mice, there were not dramatic changes in tau brain pathology. A 25-30\% reduction in phospho-tau immunostaining was observed in EpoD- vs vehicle-treated PS19 mice, but these results were statistically insignificant $(p=0.10)$, which is not unexpected as compensating for a loss of tau function may not affect tau phosphorylation and pathology (Zhang et al., 2005).

In conclusion, the results presented here provide evidence that a loss of tau function in a Tg mouse tauopathy model results in axonal dysfunction and memory impairments that can be improved by EpoD treatment. Importantly, the low doses of EpoD that elicited these beneficial effects appeared safe, and these data suggest that brain-penetrant MT-stabilizing compounds hold promise as potential therapeutic agents for the treatment of $\mathrm{AD}$ and other tauopathies.

\section{References}

Alonso AD, Zaidi T, Grundke-Iqbal I, Iqbal K (1994) Role of abnormally phosphorylated tau in the breakdown of microtubules in Alzheimer disease. Proc Natl Acad Sci U S A 91:5562-5566.

Altmann KH (2005) Recent developments in the chemical biology of epothilones. Curr Pharm Des 11:1595-1613.

Andrieux A, Salin P, Schweitzer A, Bégou M, Pachoud B, Brun P, Gory-Fauré
S, Kujala P, Suaud-Chagny MF, Höfle G, Job D (2006) Microtubule stabilizer ameliorates synaptic function and behavior in a mouse model for schizophrenia. Biol Psychiatry 60:1224-1230.

Arriagada PV, Growdon JH, Hedley-Whyte ET, Hyman BT (1992) Neurofibrillary tangles but not senile plaques parallel duration and severity of Alzheimers disease. Neurology 42:631-639.

Ballatore C, Brunden KR, Piscitelli F, James MJ, Crowe A, Yao Y, Hyde E, Trojanowski JQ, Lee VMY, Smith AB 3rd (2010) Discovery of brainpenetrant, orally bioavailable aminothienopyridazine inhibitors of tau aggregation. J Med Chem 53:3739-3747.

Bedard PL, Di Leo A, Piccart-Gebhart MJ (2010) Taxanes: optimizing adjuvant chemotherapy for early-stage breast cancer. Nat Rev Clin Oncol $7: 22-36$.

Beer TM, Higano CS, Saleh M, Dreicer R, Hudes G, Picus J, Rarick M, Fehrenbacher L, Hannah AL (2007) Phase II study of KOS-862 in patients with metastatic androgen independent prostate cancer previously treated with docetaxel. Invest New Drugs 25:565-570.

Binder LI, Frankfurter A, Rebhun LI (1985) The distribution of tau in the mammalian central nervous-system. J Cell Biol 101:1371-1378.

Dai Y, Dudek NL, Li Q, Fowler SC, Muma NA (2009) Striatal expression of a calmodulin fragment improved motor function, weight loss, and neuropathology in the R6/2 mouse model of Huntington's disease. J Neurosci 29:11550-11559.

Ertürk A, Hellal F, Enes J, Bradke F (2007) Disorganized microtubules underlie the formation of retraction bulbs and the failure of axonal regeneration. J Neurosci 27:9169-9180.

Fellner S, Bauer B, Miller DS, Schaffrik M, Fankhänel M, Spruss T, Bernhardt G, Graeff C, Färber L, Gschaidmeier H, Buschauer A, Fricker G (2002) Transport of paclitaxel (Taxol) across the blood-brain barrier in vitro and in vivo. J Clin Invest 110:1309-1318.

Goedert M (2005) Tau gene mutations and their effects. Mov Disord 20:S45-S52.

Goedert M, Jakes R, Vanmechelen E (1995) Monoclonal antibody At8 recognises tau protein phosphorylated at both serine 202 and threonine 205. Neurosci Lett 189:167-169.

Harrison FE, Hosseini AH, McDonald MP (2009) Endogenous anxiety and stress responses in water maze and Barnes maze spatial memory tasks. Behav Brain Res 198:247-251.

Hasegawa M, Smith MJ, Goedert M (1998) Tau proteins with FTDP-17 mutations have a reduced ability to promote microtubule assembly. FEBS Lett 437:207-210.

Hempen B, Brion JP (1996) Reduction of acetylated alpha-tubulin immunoreactivity in neurofibrillary tangle-bearing neurons in Alzheimer's disease. J Neuropathol Exp Neurol 55:964-972.

Hong M, Zhukareva V, Vogelsberg-Ragaglia V, Wszolek Z, Reed L, Miller BI, Geschwind DH, Bird TD, McKeel D, Goate A, Morris JC, Wilhelmsen KC, Schellenberg GD, Trojanowski JQ, Lee VMY (1998) Mutation-specific functional impairments in distinct Tau isoforms of hereditary FTDP-17. Science 282:1914-1917.

Ishihara T, Hong M, Zhang B, Nakagawa Y, Lee MK, Trojanowski JQ, Lee VMY (1999) Age-dependent emergence and progression of a tauopathy in transgenic mice overexpressing the shortest human tau isoform. Neuron 24:751-762.

Jack CR Jr, Knopman DS, Jagust WJ, Shaw LM, Aisen PS, Weiner MW, Petersen RC, Trojanowski JQ (2010) Hypothetical model of dynamic biomarkers of the Alzheimer's pathological cascade. Lancet Neurol 9:119-128.

Kidd M (1963) Paired helical filaments in electron microscopy of Alzheimers disease. Nature 197:192-193.

Laird NM, Ware JH (1982) Random-effects models for longitudinal data. Biometrics 38:963-974.

Lee CB, Wu Z, Zhang F, Chappell MD, Stachel SJ, Chou TC, Guan Y, Danishefsky SJ (2001) Insights into long-range structural effects on the stereochemistry of aldol condensations: a practical total synthesis of desoxyepothilone F. J Am Chem Soc 123:5249-5259.

Lee VMY, Goedert M, Trojanowski JQ (2001) Neurodegenerative tauopathies. Annu Rev Neurosci 24:1121-1159.

Matsuoka Y, Gray AJ, Hirata-Fukae C, Minami SS, Waterhouse EG, Mattson MP, LaFerla FM, Gozes I, Aisen PS (2007) Intranasal NAP administration reduces accumulation of amyloid peptide and tau hyperphosphorylation in a transgenic mouse model of Alzheimer's disease at early pathological stage. J Mol Neurosci 31:165-170. 
Merrick SE, Trojanowski JQ, Lee VMY (1997) Selective destruction of stable microtubules and axons by inhibitors of protein serine/threonine phosphatases in cultured human neurons (NT2N cells). J Neurosci 17:5726-5737.

O’Leary TP, Brown RE (2009) Visuo-spatial learning and memory deficits on the Barnes maze in the 16-month-old APPswe/PS1dE9 mouse model of Alzheimer's disease. Behav Brain Res 201:120-127.

Patil SS, Sunyer B, Höger H, Lubec G (2009) Evaluation of spatial memory of C57BL/6J and CD1 mice in the Barnes maze, the Multiple T-maze and in the Morris water maze. Behav Brain Res 198:58-68.

Rivkin A, Yoshimura F, Gabarda AE, Cho YS, Chou TC, Dong H, Danishefsky SJ (2004) Discovery of (E)-9,10-dehydroepothilones through chemical synthesis: on the emergence of 26-trifluoro-(E)-9,10-dehydro-12,13desoxyepothilone $\mathrm{B}$ as a promising anticancer drug candidate. J Am Chem Soc 126:10913-10922.

Wilcock GK, Esiri MM (1982) Plaques, tangles and dementia-a quantitative study. J Neurol Sci 56:343-356.
Witte H, Neukirchen D, Bradke F (2008) Microtubule stabilization specifies initial neuronal polarization. J Cell Biol 180:619-632.

Yoshiyama Y, Higuchi M, Zhang B, Huang SM, Iwata N, Saido TC, Maeda J, Suhara T, Trojanowski JQ, Lee VMY (2007) Synapse loss and microglial activation precede tangles in a P301S tauopathy mouse model. Neuron 53:337-351.

Zeger SL, Liang KY, Albert PS (1988) Models for longitudinal data- a generalized estimating equation approach. Biometrics 44:1049-1060.

Zhang B, Higuchi M, Yoshiyama Y, Ishihara T, Forman MS, Martinez D, Joyce S, Trojanowski JQ, Lee VMY (2004) Retarded axonal transport of R406W mutant tau in transgenic mice with a neurodegenerative tauopathy. J Neurosci 24:4657-4667.

Zhang B, Maiti A, Shively S, Lakhani F, McDonald-Jones G, Bruce J, Lee EB, Xie SX, Joyce S, Li C, Toleikis PM, Lee VMY, Trojanowski JQ (2005) Microtubule-binding drugs offset tau sequestration by stabilizing microtubules and reversing fast axonal transport deficits in a tauopathy model. Proc Natl Acad Sci U S A 102:227-231. 